\title{
Frictional behavior of two-dimensional materials
}

\author{
Suzhi Li ${ }^{1,2,3^{*}}$, Qunyang Lit ${ }^{4}$, Robert W. Carpick ${ }^{5}$, Peter Gumbsch ${ }^{3}$ and Ju Li ${ }^{1,2^{*}}$
}

\author{
1) Xi'an Jiaotong Univeristy, Xi'an 710049, China \\ 2) Massachusetts Institute of Technology, Cambridge, Massachusetts 02139, USA \\ 3) Karlsruhe Institute of Technology, Karlsuhe 76131, Germany \\ 4) Tsinghua University, Beijing 100084, China \\ 5) University of Pennsylvania, Philadelphia, Pennsylvania 19104, USA \\ ${ }^{*}$ E-mail lisuzhi@xjtu.edu.cn
}

Friction occurs when surfaces of solid bodies touch and move against each other. Energy is thereby converted into heat that is lost. In order to reduce the friction in the metallic elements during sliding, for example in automobiles or industrial machines, materials with a lamellar structure are commonly used as the solid lubricants. One of the dry lubricants is graphite, which has a three-dimensional layered structure.

Graphene, a two-dimensional carbon sheet, has received much attention in the past few years due to its unique mechanical, electronic properties. Earlier work has shown that the frictional behavior of graphene exhibits traits unlike those of conventional bulk materials. It has been shown experimentally that monolayer graphene shows higher friction than multilayer graphene and graphite [1]. When an atomic force microscope (AFM) tip slides on few-layer graphene loosely adhering to a substrate, the static friction force gradually strengthens for a few initial atomic periods before reaching a constant value. Such transient behavior, and the associated enhancement of steady-state friction, diminishes as the number of twodimensional layer increases. Although a wide range of suggestions have been proposed [1, 2], the mechanism behind this remains subject to debate.

Using atomistic simulations, we reproduced the experimental observations of layer dependent friction and transient frictional strengthening on graphene [3]. Atomic force analysis reveals that the evolution of static friction of graphene is strongly related to the re-adjustment of its configurations as a direct consequence of its greater flexibility. Upon sliding the tip atoms become more strongly pinned and show greater synchrony in their stick-slip motion. While the quantity of atomic-scale contacts (true contact area) evolves, the quality (in this case, the local pinning state of individual atoms and the overall commensurability) also evolves in frictional sliding on graphene.

The current findings are critical for explaining the time-dependent friction of configurationally floppy interfaces. It also suggests a means of controlling friction of twodimensional materials via strain engineering. 
(a) A tip sliding on graphene/a-Si substrate

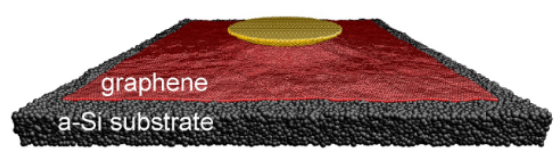

(b) Distribution of atomic friction force

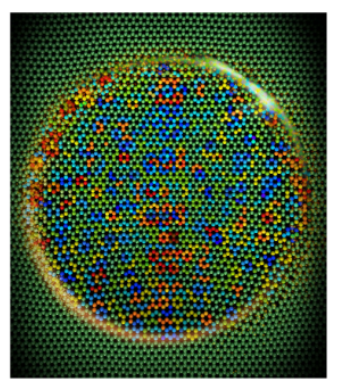

(c) Friction force trace

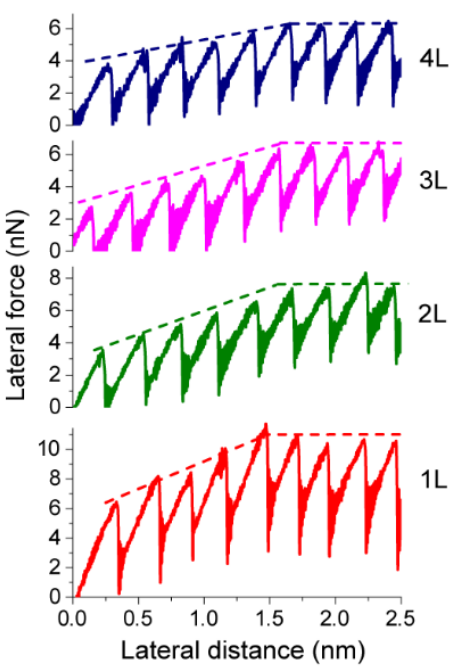

Figure 1. Frictional behavior for a Si tip sliding over a graphene/a-Si substrate system. (a) Model setup in atomistic simulations. The black, red and gold atoms refer to the a-Si substrate, graphene and the tip, respectively. (b) Illustration of distribution of atomic friction force at tip/graphene interface. (c) Friction force trace in multilayer graphene.

\section{References.}

[1] C. Lee, Q.Y. Li, W. Kalb, X.Z. Liu, H. Berger, R.W. Carpick, J. Hone, Science 328, 7680 (2010).

[2] T. Filleter, J.L. McChesney, A. Bostwick, E. Rotenberg, K. Emtsev, T. Seyller, K. Horn, R. Bennewitz, Phys. Rev. Lett. 102, 086102 (2009).

[3] S. Li, Q. Li, R.W. Carpick, P. Gumbsch, X.Z. Liu, X. Ding, J. Sun, J. Li, Nature 539, 541-545 (2016). 